\section{Consumo de dentifrício e fatores associados em um grupo populacional brasileiro}

\author{
Consumption of toothpaste and associated \\ factors in a Brazilian population group
}

\begin{abstract}
1 Universidade de Passo Fundo, Passo Fundo, Brasil.

2 Universidade Federal do Rio Grande do Sul, Porto Alegre, Brasil

Correspondência P. R. G. Colussi Universidade de Passo Fundo. BR-285, C. P. 611, Passo Fundo, RS 99052-900, Brasil. paulocolussi@upf.br
\end{abstract}

\section{Abstract}

This cross-sectional study evaluated the consumption of fluoridated dentifrice and associated factors in Passo Fundo, Rio Grande do Sul State, Brazil. A total of 688 households were selected. A structured questionnaire was answered by the mother to obtain demographics, habits, and toothpaste consumption. Household toothpaste consumption was considered low when a tube lasted $\geq 1$ month and high when $<1$ month. Logistic regression models were applied to evaluate the association between consumption and independent variables. $61.2 \%$ of households showed high toothpaste consumption. In the multivariate model, mother's age $\geq 50$ years $(\mathrm{OR}=1.62 ; 95 \% \mathrm{CI}$ : 1.02-2.61), mother's brushing frequency $(O R=$ 2.53; 95\%CI: 1.53-4.16), number of people brushing $(O R=5.69 ; 95 \% C I: 3.68-8.81)$, and cosmetic features in choice of the dentifrice $(O R=1.64$; 95\%CI: 1.03-2.61) showed the highest odds of high toothpaste consumption.

Dentifrices; Fluorine; Oral Health; Risk
Paulo Roberto Grafitti Colussi 1 Alex Nogueira Haas 2

Rui Vicente Oppermann 2

Cassiano Kuchenbecker Rösing ${ }^{2}$

\section{Introdução}

Estudos têm demonstrado o declínio da experiência de cárie no Brasil, especialmente nos últimos anos 1,2,3,4. No entanto, apesar da melhoria nos índices de cárie, a distribuição da doença ainda é desigual, sendo observada maior ocorrência especialmente entre a população mais pobre ${ }^{4,5}$.

Esta diminuição nos índices de cárie, verificada também em países desenvolvidos e em desenvolvimento, tem sido atribuída a diferentes fatores. Entre eles, destacam-se a expansão da fluoretação das águas de consumo humano, os diversos tipos de programas preventivos existentes e particularmente a disseminação do consumo de dentifrícios fluoretados. Isso fez que dentre as principais recomendações da Organização Mundial da Saúde (OMS) para melhoria da saúde bucal na população mundial esteja o uso efetivo do flúor ${ }^{6}$. Quando análises de diferentes populações são realizadas em relação ao declínio da cárie, somente um método de prevenção é comum a todos os países: dentifrício fluoretado. Isso foi observado em países que tiveram a introdução e o uso regular de creme dental fluoretado 7,8 .

Práticas de higiene bucal desempenham importante papel na prevenção às doenças bucais 9 . Sabe-se que, também, essas práticas invariavelmente incluem o uso do dentifrício. Em geral, os brasileiros citam ter hábitos razoáveis de 
higiene bucal, sobretudo em relação à frequência de escovação. A frequência de escovação mais citada é três vezes ao dia, independentemente da condição social 9,10,11. Além disso, o consumo per capita de dentifrício fluoretado tem aumentado gradativamente no Brasil, sendo considerado elevado 12,13 .

Por outro lado, o consumo exagerado de dentifrício deve ser objeto de foco da profissão, especialmente pela eventual associação do consumo inadequado com eventos adversos como, por exemplo, a fluorose dentária 12 e outros efeitos deletérios 14. Além disso, diante do quadro epidemiológico em que se observa um declínio nos índices de cárie no Brasil, mesmo que de forma desigual, aliado a um aumento expressivo no consumo per capita de dentifrício fluoretado nos últimos anos, é importante que se avalie se uma parcela da população não consome em excesso o dentifrício fluoretado e se outra parte da população não é atingida plenamente pelos benefícios de seu uso. O objetivo deste trabalho é avaliar o consumo de dentifrício fluoretado e fatores associados em um grupo populacional urbano do sul do Brasil.

\section{Material e métodos}

Este é um estudo observacional transversal de domicílios residenciais realizado na área urbana de Passo Fundo, Rio Grande do Sul, Brasil. O presente estudo foi aprovado pelo Comitê de Ética da Universidade de Passo Fundo. Todos os participantes leram e assinaram consentimento livre e esclarecido antes de integrarem o estudo.

\section{Descrição do município em estudo}

A cidade de Passo Fundo está localizada no norte do Estado do Rio Grande do Sul, distante 290km da capital, Porto Alegre. A população estimada em 2009 foi de 187.507 habitantes, com 97\% da população vivendo na zona urbana. A área territorial do município é de $780 \mathrm{~km}^{2}$, mas a área urbana possui $88 \mathrm{~km}^{2}$. O produto interno bruto (PIB) per capita de Passo Fundo em 2007 foi de $\mathrm{R} \$ 16.723,00$ (Instituto Brasileiro de Geografia e Estatística.Cidades@.http://www.ibge.gov.br/ cidadesat, acessado em Jul/2009). A expectativa de vida ao nascer é de 68,51 anos e a taxa de alfabetização, 94,8\%. A incidência de pobreza em 2003 foi de 27,91\% e o índice de Gini, 0,41. A média de moradores por domicílio é de 3,32. $\mathrm{O}$ valor do rendimento nominal médio mensal domiciliar em Passo Fundo foi de R\$ 1.416,21 15. A cidade possui fluoretação das águas de abastecimento desde 1972.

\section{Amostra}

Um total de 1.111 domicílios residenciais foi entrevistado na área urbana da cidade de Passo Fundo, que possui 49.529 domicílios particulares permanentes, dos quais 48.114 apresentam dados de rendimento domiciliar (IBGE. Censo Demográfico 2000: características da população e domicílios. http://www.ibge.gov.br, acessado em acessado em Jul/2009). O presente estudo abrangeu 2,24\% dos domicílios da cidade.

Com base no mapa da área urbana da cidade de Passo Fundo, foram selecionadas 30 áreas geográficas, de aproximadamente $3 \mathrm{~km}^{2}$. Dentro de cada área foram definidas as ruas, com suas respectivas vilas, bairros e/ou área central, a serem visitados, sempre partindo do centro da cidade em direção à periferia. Previamente, foi definido que entre 30 e 40 domicílios seriam incluídos em cada área. Se, por algum motivo, as visitas previstas para determinada rua ficassem impossibilitadas, ruas vizinhas foram selecionadas para completar o número estimado de domicílios daquela área. Caso o responsável pelo domicílio não estivesse, o mesmo era revisitado uma vez e, caso a ausência persistisse, o domicílio seguinte na mesma área era visitado. Endereços comerciais não foram contemplados no estudo. Para ser incluído no estudo, o domicílio deveria enquadrar-se em uma das três faixas de renda distintas, a saber: até 2 salários mínimos (SM), entre 5 e 7 SM e acima de 10 SM ${ }^{15}$; e a mãe ou o responsável pelo domicílio estar presente no momento da entrevista. Com base nessa metodologia de amostragem, uma amostra de conveniência de 1.111 domicílios foi obtida.

Para a análise do presente estudo, um total de 688 (61,9\%) domicílios foram incluídos. Domicílios para os quais não existiam dados referentes a variáveis a serem analisadas foram excluídos ( $315 ; 28,4 \%)$, assim como aqueles que não souberam responder à pergunta: "Quanto tempo dura um tubo de creme dental?" (108; $9,7 \%)$. A Tabela 1 mostra as características dessa amostra.

\section{Entrevista}

Os responsáveis pelos domicílios visitados foram entrevistados usando um questionário estruturado para obter dados demográficos, condição socioeconômica, hábitos e conhecimento relacionados à saúde bucal, e consumo de produtos de higiene bucal, especialmente dentifrício (que foi denominado, para fins de entendimento dos respondentes, creme dental). O questionário obteve informações sobre os hábitos dos pais e dos filhos (Tabela 1). As entrevistas foram realizadas 
Características sociodemográficas e relativas ao consumo de dentifrício da amostra em estudo. Passo Fundo, Rio Grande do Sul, Brasil, 2009.

\begin{tabular}{|c|c|c|}
\hline & $\mathrm{n}$ & $\%$ \\
\hline \multicolumn{3}{|l|}{ Idade da mãe (anos) } \\
\hline$<35$ & 153 & 22,2 \\
\hline $35-49$ & 336 & 48,9 \\
\hline$\geq 50$ & 199 & 28,9 \\
\hline \multicolumn{3}{|l|}{ Renda familiar (salários mínimos) } \\
\hline$>10$ & 102 & 14,8 \\
\hline $5-7$ & 284 & 41,3 \\
\hline$\leq 2$ & 302 & 43,9 \\
\hline \multicolumn{3}{|l|}{ Nível educacional da mãe } \\
\hline Alto & 163 & 23,4 \\
\hline Médio & 364 & 52,9 \\
\hline Baixo & 161 & 23,7 \\
\hline \multicolumn{3}{|l|}{ Assistência odontológica } \\
\hline Privada & 303 & 44,0 \\
\hline Plano de saúde & 104 & 15,1 \\
\hline Pública & 281 & 40,9 \\
\hline \multicolumn{3}{|c|}{ Frequência de escovação da mãe (vezes/dia) } \\
\hline $1-2$ & 89 & 12,9 \\
\hline$\geq 3$ & 599 & 87,1 \\
\hline \multicolumn{3}{|c|}{ Número de moradores que usam escova (moradores) } \\
\hline $1-2$ & 198 & 28,8 \\
\hline 3 & 228 & 33,1 \\
\hline$\geq 4$ & 262 & 38,1 \\
\hline \multicolumn{3}{|l|}{ Motivo da escolha do dentifrício } \\
\hline Efeito preventivo/terapêutico & 103 & 15,0 \\
\hline Outros & 585 & 85,0 \\
\hline \multicolumn{3}{|l|}{ Consumo de dentifrício } \\
\hline Baixo & 267 & 38,8 \\
\hline Alto & 421 & 61,2 \\
\hline Total & 688 & 100,0 \\
\hline
\end{tabular}

por entrevistadores treinados. Os entrevistadores receberam informações sobre os assuntos a serem pesquisados, instruções para a realização das visitas, treinamento de preenchimento do questionário antes do estudo e utilizaram procedimentos padronizados para aumentar a consistência dos resultados. Os questionários foram aplicados entre junho e julho de 2009.

\section{Análise estatística}

O domicílio foi considerado a unidade analítica do presente estudo. A análise dos dados foi realizada com uso do pacote estatístico SPSS 16 para Macintosh (SPSS Inc., Chicago, Estados Unidos) e o nível de significância foi estabelecido em $5 \%$.
O consumo de dentifrício foi considerado a variável dependente neste estudo. O consumo foi avaliado pela duração de um tubo de dentifrício aferido com as respostas dadas à pergunta "Quanto tempo dura um tubo de pasta de dente na sua casa?". Os domicílios foram categorizados em baixo (duração $\geq 1$ mês) e alto (duração $<1$ mês) consumo de dentifrício.

As variáveis independentes do presente estudo foram idade, renda, nível educacional, assistência odontológica, frequência de escovação, número de moradores e motivo da escolha do dentifrício. A renda foi avaliada utilizando-se a renda familiar do domicílio, categorizada em SM brasileiros (> $10 \mathrm{SM}$; 5-7 SM; $\leq 2 \mathrm{SM}$ ). As variáveis idade, nível educacional e frequência de escova- 
ção foram baseadas somente na mãe da família. O nível educacional foi categorizado em alto, médio e baixo de acordo com os anos de estudo da mãe ( $\geq 12$; 8-11 anos; 0-7 anos, respectivamente). O número de moradores do domicílio foi baseado apenas no número de indivíduos que utilizava escova de dente, sendo categorizado em domicílios com 1-2, 3 e $\geq 4$ moradores. Quando o dentifrício era escolhido pela presença de flúor, efeito antiplaca ou efeito antitártaro, o motivo para a compra foi categorizado em "efeito preventivo/ terapêutico". O motivo de consumo baseado no custo, preferência, propaganda ou gosto foi categorizado em "outros".

Associações entre a duração do dentifrício com as variáveis independentes foram avaliadas pelos testes qui-quadrado ou exato de Fisher, apresentadas por intermédio da distribuição de frequências. Modelos de regressão logística uni e multivariados foram utilizados para avaliar a chance de um domicílio apresentar maior consumo. Para tal, os domicílios de alto (duração $<1$ mês) foram comparados aos de baixo (duração $\geq$ 1 mês) consumo (categoria de referência).

O modelo multivariado foi construído tendo-se em conta interações e confundimento. Variáveis que apresentaram valores de $\mathrm{p}<0,25$ na análise univariada foram consideradas para o modelo multivariado. Aquelas variáveis que apresentaram associações com p > 0,10 e não alteraram estimativas de outras variáveis no modelo em $30 \%$ foram excluídas do modelo multivariado final. Não foram encontradas interações estatisticamente significantes.

\section{Resultados}

Foi observado que a maioria dos domicílios $(61,2 \%)$ apresenta alto consumo de dentifrício, caracterizado por uma duração de um tubo menor que um mês. O consumo de dentifrício considerado baixo (duração $\geq 1$ mês) foi citado por 38,8\% dos domicílios investigados (Tabela 1). A frequência de escovação três vezes (3x) ou mais foi citada por $87,1 \%$ das mães, enquanto $12,9 \%$ relataram escovar seus dentes 1 ou 2 vezes por dia (Tabela 1).

A distribuição das variáveis demográficas de acordo com o consumo de dentifrício está apresentada na Tabela 2. O consumo de dentifrício foi significativamente associado com a idade da mãe, sendo observado que quanto maior a idade da mãe da família, maior o consumo de dentifrício relatado $(\mathrm{p}<0,05)$. A renda do domicílio também apresentou associação significativa com consumo de dentifrício. Revelaram ter consumo alto de dentifrício $70,6 \%$ das famí- lias com renda acima de $10 \mathrm{SM}$, comparadas a $63,7 \%$ e 55,6\% das famílias com renda de 5-7 SM e $\leq 2 \mathrm{SM}$, respectivamente. Em relação ao nível educacional da mãe, o consumo compreendido como alto foi citado por 59,5\%, 60,4\% e $64,6 \%$ nos níveis alto, médio e baixo, respectivamente; porém sem associação significativa ( $\mathrm{p}=0,587$ ). O tipo de assistência odontológica e o motivo da escolha do dentifrício também não apresentaram associação significativa com o consumo de creme dental. Em relação aos hábitos de higiene bucal da mãe, domicílios em que a mãe relatou escovação três vezes ou mais por dia apresentaram consumo significativamente maior do que domicílios em que a mãe relatou frequência de uma a duas vezes por dia ( $p<0,001)$. Da mesma forma, o número de moradores do domicílio que usa escova de dente esteve associado com consumo de dentifrício $(\mathrm{p}<0.001)$.

A Tabela 3 mostra a análise de risco univariado da associação entre consumo de dentifrício e as diferentes variáveis independentes. Foram encontradas chances significativamente maiores de consumo alto de dentifrício no estrato intermediário de idade da mãe (35-49 anos), quando a renda familiar foi acima de $10 \mathrm{SM}$, quando a frequência de escovação da mãe foi $\geq 3 x$ /dia e em domicílios com 3 ou $\geq 4$ moradores que utilizam escova de dente. As demais variáveis estudadas (nível educacional, tipo de assistência odontológica e motivo da escolha do dentifrício) não demonstraram aumento nas chances de um consumo maior de dentifrício.

Os resultados do modelo de regressão logística multivariado são apresentados na Tabela 3 (colunas à direita). Domicílios em que a mãe possuía 50 anos ou mais de idade tiveram $62 \%$ mais chances de ter consumo alto de dentifrício do que domicílios com mães com idade abaixo de 35 anos. Renda familiar perdeu a significância da associação no modelo multivariado $(\mathrm{p}=$ 0,082 ), porém foi mantida no modelo final, pois apresentou-se como um fator de confusão, afetando a associação entre consumo e motivo da escolha do dentifrício. Escovar os dentes mais de 3 vezes ao dia aumentou significativamente as chances em 2,5 vezes. Domicílios com 3 ou mais moradores apresentaram chances significativamente maiores $(\mathrm{OR}=2,66$ e $\mathrm{OR}=5,69$ para 3 e 4 ou mais moradores, respectivamente) de alto consumo comparados a domicílios com 1-2 moradores. Também é importante ressaltar o fato de que não considerar o aspecto preventivo/terapêutico, mas sim efeitos cosméticos, propaganda e preço, na escolha do dentifrício, resultou em um aumento de $64 \%$ na chance de ter um alto consumo $(\mathrm{p}=0,038)$. 
Distribuição das variáveis demográficas e comportamentais de acordo com o consumo de dentifrício. Passo Fundo, Rio Grande do Sul, Brasil, 2009.

\begin{tabular}{|c|c|c|c|c|c|}
\hline & \multicolumn{5}{|c|}{ Consumo de dentifrício } \\
\hline & \multicolumn{2}{|c|}{ Baixo } & \multicolumn{2}{|c|}{ Alto } & \multirow[t]{2}{*}{ Valor de p * } \\
\hline & $\mathbf{n}$ & $\%$ & $\mathbf{n}$ & $\%$ & \\
\hline Idade da mãe (anos) & & & & & 0,012 \\
\hline$<35$ & 74 & 48,4 & 79 & 51,6 & \\
\hline $35-49$ & 115 & 34,2 & 221 & 65,8 & \\
\hline$\geq 50$ & 78 & 39,2 & 121 & 60,8 & \\
\hline Renda familiar (salários mínimos) & & & & & 0,014 \\
\hline$>10$ & 30 & 29,6 & 72 & 70,6 & \\
\hline $5-7$ & 103 & 36,3 & 181 & 63,7 & \\
\hline$\leq 2$ & 134 & 44,4 & 168 & 55,6 & \\
\hline Nível educacional da mãe & & & & & 0,587 \\
\hline Alto & 66 & 40,5 & 97 & 59,5 & \\
\hline Médio & 144 & 39,6 & 220 & 60,4 & \\
\hline Baixo & 57 & 35,4 & 104 & 64,6 & \\
\hline Assistência odontológica & & & & & 0,428 \\
\hline Privada & 113 & 37,3 & 190 & 62,7 & \\
\hline Plano de saúde & 37 & 35,6 & 67 & 64,4 & \\
\hline Pública & 117 & 41,6 & 164 & 58,4 & \\
\hline Frequência escovação da mãe (vezes/dia) & & & & & $<0,001 \star \star$ \\
\hline $1-2$ & 53 & 59,6 & 36 & 40,4 & \\
\hline$\geq 3$ & 214 & 35,7 & 385 & 64,3 & \\
\hline Número de moradores que usam escova (moradores) & & & & & $<0,001$ \\
\hline $1-2$ & 123 & 62,1 & 75 & 37,9 & \\
\hline 3 & 86 & 37,7 & 142 & 62,3 & \\
\hline$\geq 4$ & 58 & 22,1 & 204 & 77,9 & \\
\hline Motivo da escolha do dentifrício & & & & & 0,08 ** \\
\hline Efeito preventivo/terapêutico & 48 & 46,6 & 55 & 53,4 & \\
\hline Outros & 219 & 37,4 & 366 & 62,6 & \\
\hline
\end{tabular}

* Qui-quadrado;

** Teste exato de Fisher.

\section{Discussão}

O presente estudo procurou avaliar consumo de dentifrício e fatores associados em uma cidade brasileira de porte médio do sul do Brasil. O estudo do consumo de dentifrícios é de extrema importância, na medida em que, por um lado está associado à diminuição dos índices de cárie e melhora da saúde bucal, e por outro, existem riscos de seu uso excessivo. Da mesma forma, por se tratar de um bem de consumo, o estudo de fatores demográficos e socioeconômico-culturais é imperioso. Nesse sentido, os achados deste estudo evidenciaram que a maioria dos domicílios investigados possui alto consumo de dentifrício, com duração de um tubo menor do que um mês.
Além disso, os fatores associados ao maior consumo de dentifrício foram idade da mãe acima de 50 anos, alta frequência de escovação, maior número de moradores que usam escova de dente e apelo comercial e cosmético do dentifrício. Não foram observados moradores que utilizam dentifrícios desvinculados do uso de escova de dente.

Um dos achados do presente estudo está vinculado ao fato de que, quanto maior a idade da mãe, maior o consumo de dentifrício do domicílio. Uma das possíveis explicações para esta associação pode ser vinculada às práticas mais antigas de higiene bucal (provavelmente repassadas por essas mães) que sugeriam consumo de quantidades maiores de dentifrício para que se obtivesse o seu melhor efeito. Mais recente- 
Modelos de regressão logística ajustado e não ajustado da associação de variáveis independentes com o consumo de dentifrício (referência: baixo consumo). Passo Fundo, Rio Grande do Sul, Brasil, 2009.

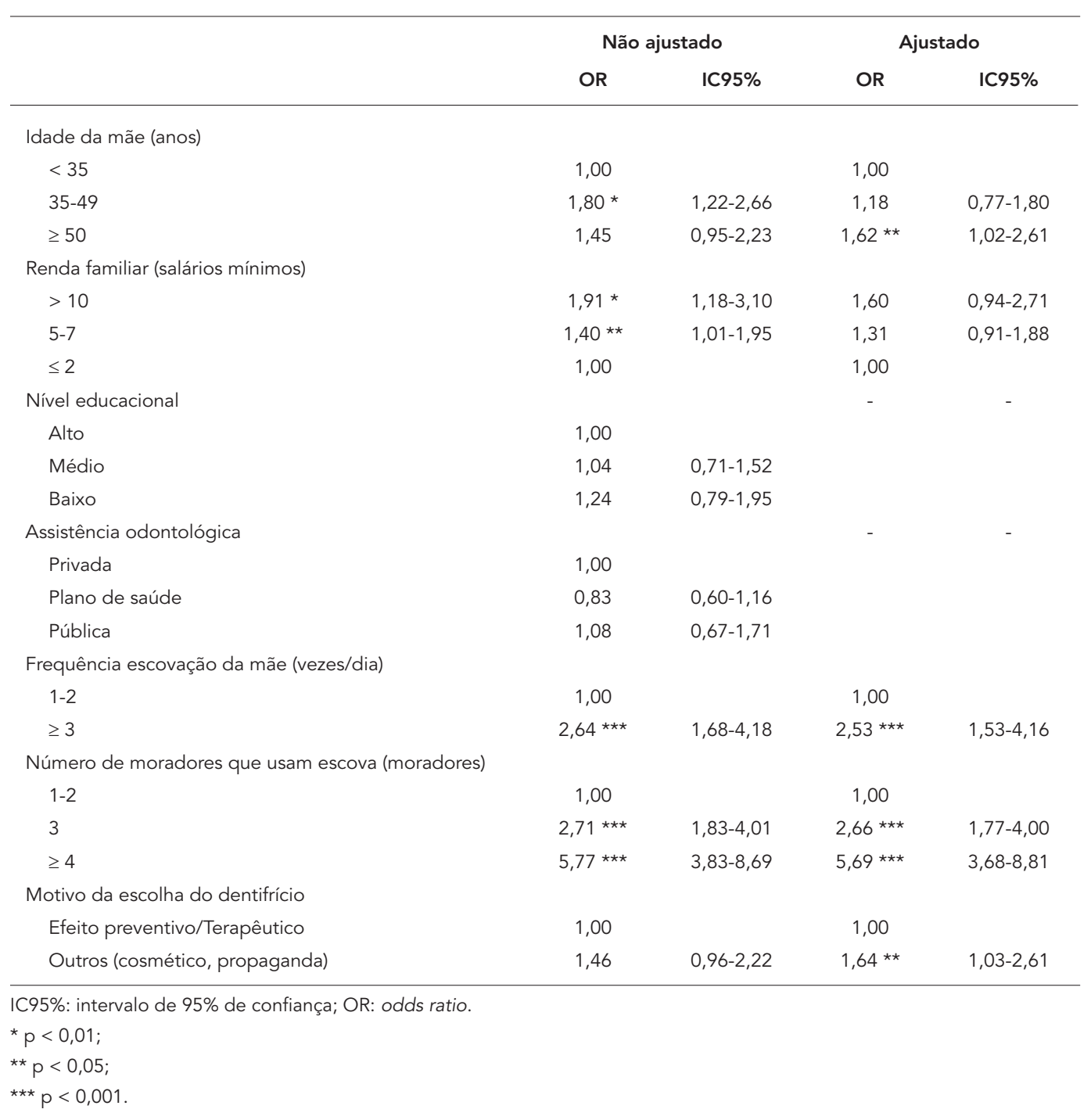

mente, houve uma mudança de conduta frente à quantidade de dentifrício necessário para realizar a escovação, além disso, também tem sido discutida a concentração de flúor nos dentifrícios, sobretudo com o intuito de diminuir os riscos à fluorose dentária 16 .

Tem sido relatada, em outros países, uma associação entre renda e uso de dentifrício 17,18. Um estudo na Tanzânia demonstrou que a maioria dos indivíduos que usava dentifrício menos do que uma vez ao dia justificou sua conduta por motivos financeiros 18. Nesta amostra brasileira, o consumo de dentifrício foi significativamente maior em famílias de maior renda, e a chance de maior consumo também foi significativamen- te maior nos domicílios de alta renda na análise univariada. Contudo, renda familiar perdeu a significância estatística na análise multivariada, permanecendo no modelo por apresentar-se como um confundidor da associação entre consumo e motivo da escolha do dentifrício. Isso pode indicar que famílias de maior renda priorizam o consumo do dentifrício não somente pelos seus efeitos preventivo/terapêuticos, mas especialmente pelo apelo da propaganda e efeito cosmético dos produtos de higiene bucal. No Japão, foi demonstrado que a maioria dos indivíduos consome dentifrício sem dar atenção especial a seus benefícios preventivos 19. Parece que, no Brasil, o consumo de produtos de saúde tem sido priori- 
zado em famílias com maiores condições financeiras, apontando para o papel que a propaganda e o apelo cosmético têm tido no consumo de bens de saúde. Por outro lado, a informação mais prevalente em relação a número de escovações ao dia foi de três vezes. É possível que essa resposta seja influenciada pela convenção social de que essa é a frequência recomendada no Brasil. Possivelmente em países onde a recomendação hegemônica seja de duas vezes ao dia, essa seria a resposta mais frequente. Entretanto, o elevado consumo brasileiro per capita de dentifrício faz supor que o brasileiro, em média, escove os seus dentes frequentemente.

O nível educacional da mãe tem sido considerado um preditor de cárie, assim como influencia os hábitos de higiene bucal dos filhos 20,21,22. Contudo, na análise univariada realizada no presente estudo, diferenças no consumo de dentifrício entre os estratos de nível educacional não foram encontradas. Por outro lado, sabe-se que mulheres apresentam hábitos de higiene bucal mais adequados se comparadas aos homens 23 . No presente estudo, um reflexo indireto disso foi o achado de que o número de escovações da mãe foi um fator significativamente associado ao maior consumo de dentifrício, indicando um papel disseminador dos hábitos da mãe nos hábitos da família. De certa forma, esse era um achado esperado, pois quem escova mais seus dentes deverá comprar mais dentifrício.

Quanto à frequência de escovação, deve-se salientar que escovação dos dentes três vezes ou mais ao dia foi reportada pela maioria dos domicílios $(87,1 \%)$. Tal frequência é semelhante a outros estudos feitos no Brasil 9,10,11 e diferente dos estudos realizados em outros países, onde a frequência preponderante é uma ou duas vezes ao dia 24,25 .

Os achados do presente estudo, utilizando o domicilio como unidade analítica, podem ajudar a entender o consumo familiar de dentifrício no Brasil. Nesse sentido, um achado do presente estudo foi que quanto maior o número de moradores do domicílio, maior a chance de ter alto consumo. De maneira geral, os estudos sobre hábitos de higiene bucal têm focado a frequência de escovação ou o consumo per capita de creme dental (obtido pela média de consumo por indivíduo-usuário do produto), e não no consumo individual (referido pelo indivíduo que usa o produto sem compartilhá-lo) ou domiciliar. Isso implica que não se conheçam os extremos do consumo em uma população que já apresenta um consumo considerado elevado, maior que muitos países desenvolvidos. De qualquer forma, na ausência de dados sobre higiene bucal, os dados descrevendo venda de produtos de higiene bucal podem ser utilizados como um indicador de hábitos de higiene bucal em uma população 13 . É importante que se tenha em mente que a variável dependente deste estudo - consumo de dentifrício por domicílio - é sujeita a limitações e a sua definição em termos de ponto de corte se deu pela distribuição dos dados, sendo avaliado como alto o consumo de mais de um tubo de dentifrício por mês e baixo quando esse consumo foi menor que um tubo por mês. Como a unidade de pesquisa foi o domicílio e não o indivíduo, uma vez que uma entrevista foi realizada por domicílio, isso poderia gerar um viés de classificação. Entretanto, para diminuir os efeitos negativos dessa sabida limitação, o número de indivíduos que usa escova de dente por domicílio foi incluído como variável de ajuste no modelo final. Também é importante ressaltar que a prática de compra de dentifrício está normalmente vinculada ao consumo do domicílio, uma vez que não se considera o dentifrício um bem de consumo individual.

A amostra do presente estudo foi derivada de uma sistemática que buscou representar os estratos socioeconômicos da cidade de Passo Fundo. Todavia, deve-se salientar que esta amostra não deve ser considerada representativa dessa cidade, pois nenhum método aleatório de amostragem foi aplicado. Mesmo assim, o percentual de domicílios com renda de até 2 SM foi semelhante ao reportado pelo IBGE no último censo realizado na região (Censo Demográfico 2000. http://www.ibge.gov.br). Além disso, o tamanho da amostra do presente estudo é semelhante a diversos estudos que abordam hábitos de higiene bucal de cidades de porte maior 9,26 e de cidades de porte semelhante ao de Passo Fundo 22,25.

O uso efetivo do flúor, recomendação prioritária da OMS para controle da cárie dentária 6, deve ser foco de atenção da Odontologia. Dentre os aspectos que deveriam ser mais estudados é o aumento gradativo no consumo, verificado nos últimos anos. Aspectos como a estabilização econômica e o controle da inflação, bem como a melhora na distribuição de renda, verificados nos últimos anos, deveriam ser discutidos quando se observa o consumo elevado de dentifrício, a diminuição na prevalência de cárie e a ocorrência maior da doença na camada mais pobre da população.

Por fim, pode-se concluir que o alto consumo de dentifrícios é elevado na amostra em estudo, e idade da mãe, frequência de escovação da mãe, número de moradores que usam escova de dentes e motivo da escolha do dentifrício estiveram associados a um maior consumo dos mesmos. 


\section{Resumo}

Este estudo transversal avaliou o consumo de dentifrício fluoretado e fatores associados em Passo Fundo, Rio Grande do Sul, Brasil. Seiscentos e oitenta e oito domicílios foram selecionados. Um questionário estruturado foi respondido pela mãe para obter dados demográficos, hábitos e consumo de dentifrício. O consumo foi avaliado por intermédio da duração de um tubo de dentifrício e considerado baixo (duração $\geq 1$ mês) e alto (duração < 1 mês). Modelos de regressão logística avaliaram a associação do consumo com variáveis independentes. Foi observado que 61,2\% dos domicílios apresentaram alto consumo de dentifrício. No modelo multivariado, idade da mãe $\geq 50$ anos (OR = 1.62; IC95\% 1,02-2,61), frequência de escovação da mãe (OR = 2,53; IC95\%: 1,53-4,16), número de moradores que utilizam escova (OR = 5,69; IC95\%: 3,68-8,81) e motivo cosmético para escolha do dentifrício $(\mathrm{OR}=$ 1,64; IC95\%:1,03-2,61) representaram maior chance de alto consumo de dentifrício. Conclui-se que a maioria dos domicílios apresenta consumo elevado de dentifrício, associado com idade e frequência de escovação da mãe, número de moradores e motivos de escolha.

Dentifrício; Flúor; Saúde Bucal; Risco

\section{Referências}

1. Narvai PC, Frazão P, Roncalli AG, Antunes JLF. Cárie dentária no Brasil: declínio, polarização, iniqüidade e exclusão social. Rev Panam Salud Pública 2006; 19:385-93.

2. Peres MA, Oliveira-Latorre MR, Sheiham A, Peres KG, Barros FC, Hernandes PG, et al. Social and biological early life influences on severity of dental caries in children aged 6 years. Community Dent Oral Epidemiol 2005; 33: 53-63.

3. Carvalho JC, Figueiredo MJ, Vieira EO, Mestrinho HD. Caries trends in Brazilian non-privileged preschool children in 1996 and 2006. Caries Res 2009; 43:2-9.

4. Tagliaferro EPS, Meneghim MC, Ambrosano GMB, Pereira AC. Distribution and prevalence of dental caries in Bauru, Brazil, 1976-2006. Int Dent J 2008; 58:75-80.

5. Peres SHCS, Carvalho FS, Carvalho CP, Bastos JRM, Lauris JRP. Polarização da cárie dentária em adolescentes, na região sudoeste do Estado de São Paulo, Brasil. Ciênc Saúde Coletiva 2008; 13:2155-62.

\section{Colaboradores}

P. R. G. Colussi foi o responsável pela construção e execução do projeto, coleta de dados e participou ativamente na análise dos dados e redação do manuscrito. A. N. Haas orientou a construção do projeto, realizou a análise dos dados e a redação do manuscrito. R. V. Oppermann participou da construção do projeto, da interpretação dos achados e redação do manuscrito. C. K. Rösing, idealizador do projeto, participou da construção, interpretação dos dados e redação do manuscrito.

\section{Agradecimentos}

Os autores gostariam de agradecer à professora Nayme Marlene Nemenn da Silva e aos alunos da Faculdade de Odontologia da Universidade de Passo Fundo que participaram da coleta dos dados.
6. Petersen PE. Global policy for improvement of oral health in the 21st century - implications to health research of World Health Assembly 2007, World Health Organization. Community Dent Oral Epidemiol 2009; 37:1-8.

7. Nadanovsky P, Sheiham A. Relative contribution of dental services to the changes in caries levels of 12-year-old children in 18 industrialized countries in the 1970s and early 1980s. Community Dent Oral Epidemiol 1995; 23:331-9.

8. Jackson RJ, Newman HN, Smart GJ, Stokes E, Hogan JI, Brown C, et al. The effects of a supervised toothbrushing programme on the caries increment of primary school children, initially aged 5-6 years. Caries Res 2005; 39:108-15.

9. Abegg C. Hábitos de higiene bucal de adultos porto-alegrenses. Rev Saúde Pública 1997; 31:586-93.

10. Colussi PRG. Perfil de consumo de produtos de higiene bucal em Passo Fundo/RS [Dissertação de Mestrado]. Canoas: Faculdade de Odontologia, Universidade Luterana do Brasil; 1998. 
11. Freddo SL, Aerts DRGC, Abbeg C, Davoglio R, Vieira PC, Monteiro L. Hábitos de higiene bucal e utilização de serviços odontológicos em escolares de uma cidade da região Sul do Brasil. Cad Saúde Pública 2008; 24:1991-2000.

12. Lima YBO, Cury JA. Ingestão de flúor por crianças pela água e dentifrício. Rev Saúde Pública 2001; 35:576-81.

13. Gjermo P, Rösing CK, Susin C, Oppermann RV. Periodontal diseases in Central and South America. Periodontology 2000 2002; 29:70-8.

14. West NX, Moran JM. Home-use preventive and therapeutic oral products. Periodontology 2000 2008; 48:7-9.

15. Instituto Brasileiro de Geografia e Estatística. Mapa de pobreza e desigualdade - municípios brasileiros 2003 [DVD]. Rio de Janeiro: Instituto Brasileiro de Geografia e Estatística; 2003.

16. Lima TJ, Ribeiro CCC, Tenuta LMA, Cury JA. Lowfluoride dentifrice and caries lesion control in children with different caries experience: a randomized clinical trial. Caries Res 2008; 42:46-50.

17. Liu M, Zhu L, Zhang B, Petersen PE. Changing use and knowledge of fluoride toothpaste by schoolchildren, parents and schoolteachers in Beijing, China. Int Dent J 2007; 57:187-94.

18. Kikwilu EN, Frencken JE, Mulder J. Utilization of toothpaste and fluoride content in toothpaste manufactured in Tanzania. Acta Odontol Scand 2008; 66:293-99.

19. Fukuda H, Shinsho F, Shigemassa A, Saito T. Relationships between dental health behaviour of japanese school children and their parents. Int Dent J 2007; 57:319-23.
20. Perera I, Ekanayake L. Factors influencing perception of oral health among adolescents in Sri Lanka. Int Dent J 2008; 58:349-55.

21. Grembowski D, Spiekerman C, Milgrom P. Linking mother access to dental care and child oral health. Community Dent Oral Epidemiol 2009; 37:381-90.

22. Noro LRA, Roncalli AG, Mendes Júnior FIR, Lima KC. Incidência de cárie dentária em adolescentes em município do nordeste brasileiro, 2006. Cad Saúde Pública 2009; 25:783-90.

23. Harada S, Akhter R, Kurita K, Mori M, Hoshikshi M, Tamashiro H, et al. Relationships between lifestyle and dental health behaviors in a rural population in Japan. Community Dent Oral Epidemiol 2005; 33:17-24.

24. Kalsbeek H, Truin GJ, Poorteman JHG, van Rossum GMJM, van Rijkom HM, Verrips GHW. Trends in periodontal status and oral hygiene habits in Dutch adults between 1983 and 1995. Community Dent Oral Epidemiol: 2000; 28:112-8.

25. Hugoson A, Koch G, Göthberg C, Helkimo AN, Lundin S, Norderyd O, et al. Oral health of individuals Aged 3-80 years in Jönköping, Sweden during 30 years (1973-2003). Swed Dent J 2005; 29:125-38.

26. Grindefjord M, Dahllöf G, Nilsson B. Stepwise prediction of dental caries in children up to 3.5 years of age. Caries Res 1996; 30:256-66.

Recebido em 15/Jun/2010

Versão final reapresentada em 10/Nov/2010 Aprovado em 17/Jan/2011 